\title{
Staphylococcus lentus
}

National Cancer Institute

\section{Source}

National Cancer Institute. Staphylococcus lentus. NCI Thesaurus. Code C86766.

A species of facultatively anaerobic, Gram positive, cocci shaped bacteria in the phylum Firmicutes. This species is positive for catalase, oxidase and caseinase and neg ative for urease, and coagulase, It can ferment glucose, fructose, ribose, cellobiose, mannitol, mannose, sucrose, and trehalose, but not xylose, turanose, or xylitol. S. lentus is commensal with humans and many other animals and is an opportunistic pathogen in its hosts. 